\title{
Topological Anderson insulator phase in a Dirac-semimetal thin film
}

\author{
Rui Chen, Dong-Hui Xu*, and Bin Zhou ${ }^{\dagger}$ \\ Department of Physics, Hubei University, Wuhan 430062, China
}

(Dated: May 25, 2017)

\begin{abstract}
The recently discovered topological Dirac semimetal represents a new exotic quantum state of matter. Topological Dirac semimetals can be viewed as three dimensional analogues of graphene, in which the Dirac nodes are protected by crystalline symmetry. It has been found that quantum confinement effect can gap out Dirac nodes and convert Dirac semimetal to a band insulator. The band insulator is either normal insulator or quantum spin Hall insulator depending on the thin film thickness. We present the study of disorder effects in thin film of Dirac semimetals. It is found that moderate Anderson disorder strength can drive a topological phase transition from normal band insulator to topological Anderson insulator in Dirac semimetal thin film. The numerical calculation based on the model parameters of Dirac semimetal $\mathrm{Na}_{3} \mathrm{Bi}$ shows that in the topological Anderson insulator phase a quantized conductance plateau occurs in the bulk gap of band insulator, and the distributions of local currents further confirm that the quantized conductance plateau arises from the helical edge states induced by disorder. Finally, an effective medium theory based on Born approximation fits the numerical data.
\end{abstract}

PACS numbers: 73.20.-r, 73.20.Fz, 73.43.Nq, 03.65.Vf

\section{INTRODUCTION}

Two-dimensional (2D) massless Dirac fermions have been observed in graphene and on the surface of threedimensional (3D) topological insulators (TIs) $\underline{\underline{1}} \underline{\underline{\underline{6}}} \mathrm{Re}-$ cently, much attention has been attracted to the Dirac semimetal which represents a new state of quantum matter, can support 3D Dirac fermions with the linear dispersion in all three momentum directions. The stable Dirac semimetals have been realized experimentally in two crystalline materials, $\mathrm{Na}_{3} \mathrm{Bi}$ and $\mathrm{Cd}_{3} \mathrm{As}_{2}$ compounds ${ }^{7-11}$ The stability of the 3D Dirac points in these materials requires additional crystalline symmetries other than the time-reversal symmetry and inversion symmetry 12 Dirac semimetals show many interesting physics properties, such as spin polarized double Fermi arc surface states and chiral anomaly in the presence of parallel electric and magnetic fields $\underline{13,14}$

Finite size effect plays an important role in the system with size comparable to the quasiparticle wavelength. In 2DTIs, the states on the opposite edges can couple with each other to open a gap in the gapless edge energy spectrum due to finite size effect. $\frac{15}{5}$ While for the thin film of 3DTIs, depending on the thickness, it may become a quantum spin Hall (QSH) insulator or a trivial band insulator $\stackrel{16}{-20}$ Similar properties are also proposed in Dirac semimetal materials $\mathrm{Na}_{3} \mathrm{Bi}$ and $\mathrm{Cd}_{3} \mathrm{As}_{2}$, in which the quantum confinement effect can induce a periodically modulated gap at Dirac node $\frac{7,8,21,22}{2}$ Whether a finite size gap is inverted or not is determined by the thickness of Dirac semimetal thin film. Thus, Dirac semimet-

\footnotetext{
*donghuixu@hubu.edu.cn

†binzhou@hubu.edu.cn
}

als $\mathrm{Na}_{3} \mathrm{Bi}$ and $\mathrm{Cd}_{3} \mathrm{As}_{2}$ should cross over between trivial and nontrivial 2D insulators oscillatorily as a function of Dirac semimetal thin film thickness $\frac{7.8}{2}$

It is well known that disorder has a major impact upon the transport properties of low-dimensional electronic systems ${ }^{23}$ In 2009 , Li et al. reported that the onsite Anderson type disorder can trigger a phase transition from topologically trivial phase to QSH phase with quantized edge conductance $\stackrel{24}{=}$ This disorder-induced topological insulator was named as topological Anderson insula-

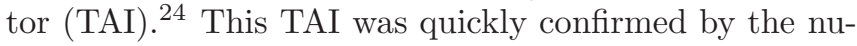
merical simulations and Born approximation analysis in which the disorder-induced topological phase transition can be understood by the renormalization of Dirac mass and chemical potential. $\stackrel{25,26}{ }$ TAI has been investigated in more related models, such as Haldane model, KaneMele model and 3DTI model ${ }^{27-32}$ It is indicated that TAI is dependent on the type of disorder, for example, TAI phase is absent for the magnetic disorder and spatially correlated disorder $\stackrel{33.34}{ }$ On the other hand, gapped and gapless topological phases and transport characteristics in layered structures and thin films have been studied recently in the presence and absence of disorder $\stackrel{35-37}{-3 t}$ is interesting to note that Kobayashi et al. revealed the TAI phase in topological insulator nanofilms by numerical calculations $\underline{\underline{36}}$

In this paper, we investigate the interplay of disorder and quantum confinement in Dirac semimetal thin film. It is found that the Anderson disorder can induce a topological phase transition in this system. Topological Anderson insulating phase emerges by tuning the strength of disorder. By combining numerical simulation based on the recursive Green's function and Born approximation with the model parameters of Dirac semimetal $\mathrm{Na}_{3} \mathrm{Bi}$, we show that when the on-site disorder is introduced to the insulating phase of Dirac semimetal with spin Chern 
number $C_{s}=0$, the TAI phase with a quantized conductance plateau $\left(\sigma=2 e^{2} / h\right)$ appears in a certain range of disorder strength. Inspection of the nonequilibrium local current distribution further confirms that this quantized conductance plateau arises from the helical edge states induced by disorder.

\section{MODEL}

We start with a generic low-energy effective model derived from first principles results of Dirac semimetals $\mathrm{A}_{3} \mathrm{Bi}(\mathrm{A}=\mathrm{Na}, \mathrm{K}, \mathrm{Rb})$ and $\mathrm{Cd}_{3} \mathrm{As}_{2}{ }_{2} \underline{\underline{7}}, \underline{8}$ In the basis of $\left|S_{\frac{1}{2}}, \frac{1}{2}\right\rangle,\left|P_{\frac{3}{2}}, \frac{3}{2}\right\rangle,\left|S_{\frac{1}{2}},-\frac{1}{2}\right\rangle,\left|P_{\frac{3}{2}},-\frac{3}{2}\right\rangle$, the Hamiltonian of effective model can be expressed as

$$
H(\mathbf{k})=\epsilon_{0}(\mathbf{k})+\left(\begin{array}{cccc}
M(\mathbf{k}) & A k_{+} & 0 & 0 \\
A k_{-} & -M(\mathbf{k}) & 0 & 0 \\
0 & 0 & M(\mathbf{k}) & -A k_{-} \\
0 & 0 & -A k_{+} & -M(\mathbf{k})
\end{array}\right)
$$

where $\left|S_{\frac{1}{2}}, \pm \frac{1}{2}\right\rangle$ are the conduction $s$ state and $\left|P_{\frac{3}{2}}, \pm \frac{3}{2}\right\rangle$ are the heavy-hole $p$ state, $\epsilon_{0}(\mathbf{k})=C_{0}+C_{1} k_{z}^{2}+C_{2} k_{\|}^{2}$, $M(\mathbf{k})=-M_{0}+M_{1} k_{z}^{2}+M_{2} k_{\|}^{2}, \mathbf{k}_{\|}=\left(k_{x}, k_{y}\right)$, and $k_{ \pm}=$ $k_{x} \pm i k_{y}$. There are two Dirac nodes located at $\left(0,0, \pm k_{D}\right)$ with $k_{D}=\sqrt{M_{0} / M_{1}}$.

In the following calculations, we will take the model parameters of Dirac semimetal $\mathrm{Na}_{3} \mathrm{Bi}$ obtained from the first-principles calculations, $\underset{-1}{-}$ namely $C_{0}=-63.82 \mathrm{meV}$, $C_{1}=87.538 \mathrm{meV} \cdot \mathrm{nm}^{2}, C_{2}=-84.008 \mathrm{meV} \cdot \mathrm{nm}^{2}, M_{0}=$ $86.86 \mathrm{meV}, M_{1}=106.424 \mathrm{meV} \cdot \mathrm{nm}^{2}, M_{2}=103.610$ $\mathrm{meV} \cdot \mathrm{nm}^{2}$, and $A=245.98 \mathrm{meV} \cdot \mathrm{nm}$. We discretize the effective Hamiltonian on a $3 \mathrm{D}$ simple cubic lattice and set the lattice constants as $a_{x}=a_{y}=a_{z}=a=0.5 \mathrm{~nm}$.
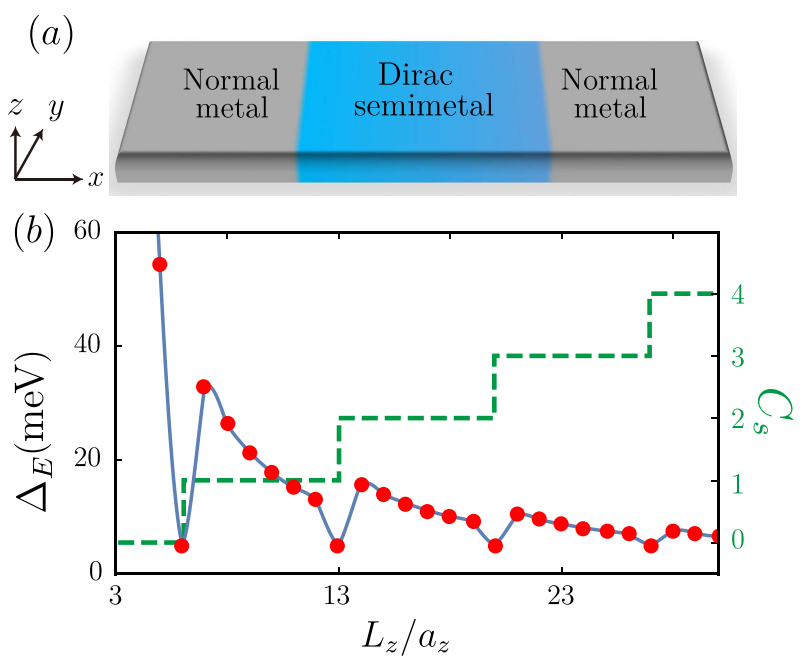

FIG. 1: (Color online) (a) Schematic illustration of metalDirac semimetal-metal setup. (b) Confinement-induced bulk gap $\Delta_{E}$ (blue solid line with red dots) and the spin Chern number $C_{s}$ (green dashed line) versus thin film thickness $L_{z}$.
Now let us consider a thin film of Dirac semimetal confined along $z$ direction with thickness $L_{z}$. Due to quantum confinement effect, the energy band along $z$ direction will be quantized into separated levels. The confinementinduced band gap as a function of thickness is presented in Fig. 1(b). Using quantum well approximation, the following term can be obtained for each subband $n^{21,22}$

$$
M(\mathbf{k}) \rightarrow M\left(n, \mathbf{k}_{\|}\right)=\mathcal{M}_{n}+M_{2} k_{\|}^{2},
$$

where $\mathcal{M}_{n}=-M_{0}+M_{1}\left(n \pi / L_{z}\right)^{2}$ is the mass parameter of these subbands. For each subband $n$, the Hamiltonian (11) is similar to the BHZ model Hamiltonian describing the QSH system in $\mathrm{HgTe} / \mathrm{CdTe}$ quantum wells 38

To characterize the system, we will calculate the spin Chern number $C_{s}$ within quantum well approximation ${ }^{39-41}$ It has been shown that the spin Chern number and $Z_{2}$ topological invariant would yield the same classification by investigating topological properties of time-reversal invariant systems. 42 The spin Chern number is defined as 43

$$
C_{s}=\frac{1}{2} \sum_{n}\left(C_{\uparrow}^{n}-C_{\downarrow}^{n}\right),
$$

where $C_{\uparrow}^{n}$ and $C_{\downarrow}^{n}$ are the valence band Chern number of the spin up and down blocks of the $n$-th subband, respectively. The results are shown in Fig. 1(b), in which the green dashed line depicts the spin Chern number. When the thickness of this system is small $\left(L_{z}<6 a_{z}\right)$, the spin Chern number keeps zero, indicating that the system is actually a normal insulator. While if the thickness exceeds a critical value $\left(L_{z} \approx 6 a_{z}\right)$, the energy gap closes and reopens, and a nonzero spin Chern number $C_{s}=1$ is obtained, which means that a topological phase transition from the normal insulator to the QSH state has happened. With further increasing $L_{z}$, this system shows more than one Kramer's pair of helical edge states, characterized by higher spin Chern numbers with $C_{s}=2,3, \cdots$. The spin Chern number is exactly equal to the number of the pair of one-way helical edge states. The odd spin Chern number corresponds to the QSH phase, while the even spin Chern number corresponds to the normal insulator. Thus this system crosses over between trivial and nontrivial 2D insulators oscillatorily as a function of thin film thickness. The result given by the spin Chern number is consistent with the previous results $, 7,8$

To better understand the different phase in Dirac semimetal thin film tuned by finite size effect, we plot the energy band spectrum for thickness $L_{z}=7 a_{z}$ and $L_{z}=5 a_{z}$ in Fig. 2(a) and (d), which correspond to the QSH state and normal insulating state, respectively. For the case of $L_{z}=5 a_{z}$, since all the subbands are trivial, there is no edge state on the side surfaces of Dirac semimetal. While for $L_{z}=7 a_{z}$, one subband becomes inverted, as a result, there is a pair of helical edge states on the side surfaces, as indicated by the red curves. For a thicker sample with $L_{z}=15 a_{z}$, there will exit two pairs 

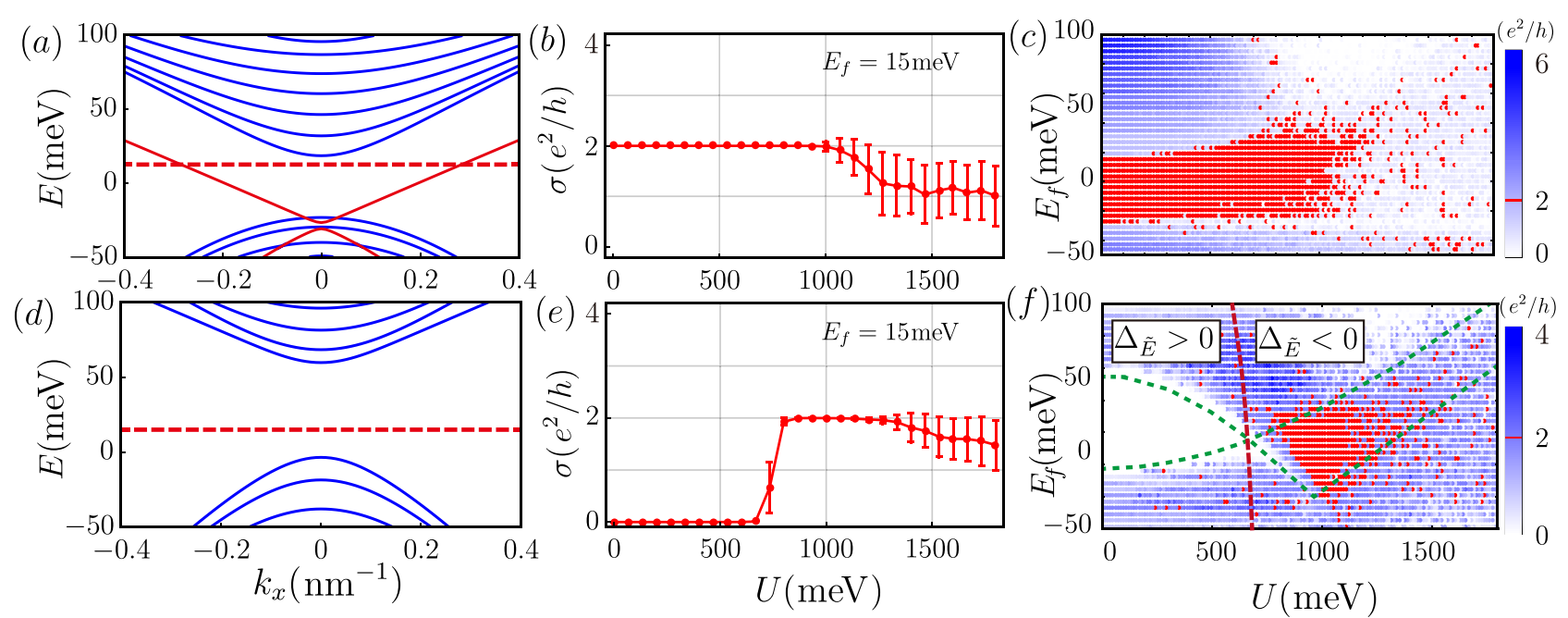

FIG. 2: (Color online) (a) Energy spectrum of Dirac semimetal thin film with open boundary conditions along $y$ and $z$ directions and periodic boundary condition along $x$ direction. Here, the sizes in the $y$ and $z$ directions are taken as $L_{y}=80 a_{y}$ and $L_{z}=7 a_{z}$, respectively. The blue curves are the bulk subbands and the red lines correspond to the surface states. (b) The disorder averaged conductance as a function of disorder strength $U$. The error bars show standard deviation of the conductance for 500 samples. The Fermi level is $E_{f}=15 \mathrm{meV}$. (c) Phase diagram showing the conductance as a function of disorder strength $U$ and Fermi energy $E_{f}$. Each data point corresponds to a single realization of the disorder potential. The data points marked in red represent the value of conductance within the interval $(2-\epsilon) e^{2} / h<\sigma<(2+\epsilon) e^{2} / h$, and we take $\epsilon=0.005$. The other panels (d), (e) and (f) are the same with (a), (b) and (c), except that $L_{z}=5 a_{z}$. The red and green dashed lines in (f) correspond to the phase boundary defined as $\Delta_{\tilde{E}}=0$ and $\tilde{E}_{2}<\tilde{E}_{f}<\tilde{E}_{1}$. In our numerical simulations, the system size is chosen as $L_{z}=7 a_{z}, L_{y}=80 a_{y}$ and $L_{x}=300 a_{x}$ in (b) and (e), and $L_{z}=5 a_{z}, L_{y}=80 a_{y}$ and $L_{x}=150 a_{x}$ in (c) and (f).

of helical edge states, corresponding to the spin Chern number $C_{s}=2$.

\section{NUMERICAL SIMULATION}

Now, we will investigate the transport properties of the Dirac semimetal thin film in the presence of disorder by using the Landauer-Büttiker-Fisher-Lee formula $\underline{44}-\underline{46}$ and the recursive Green's function method $\underline{47}, \underline{48}$ The linear conductance can be obtained by $\sigma=\left(e^{2} / h\right) T$, where $T=\operatorname{Tr}\left[\Gamma_{L} G^{r} \Gamma_{R} G^{a}\right]$ is the transmission coefficient. The linewidth function $\Gamma_{\alpha}\left(E_{f}\right)=i\left[\Sigma_{\alpha}^{r}-\Sigma_{\alpha}^{a}\right]$ with $\alpha=L, R$, and the Green's functions $G^{r / a}\left(E_{f}\right)$ are calculated from $G^{r}\left(E_{f}\right)=\left[G^{a}\left(E_{f}\right)\right]^{\dagger}=\left[E I-H_{C}-\Sigma_{L}^{r}-\Sigma_{R}^{r}\right]^{-1}$, where $E_{f}$ is the Fermi level, $H_{C}$ is the Hamiltonian matrix of the central scattering region, and $\Sigma_{L, R}^{(r / a)}$ are the retarded (advanced) self-energy due to the device leads.

We adopt a rectangular thin film sample of size $L_{x} \times$ $L_{y} \times L_{z}$ and two semi-infinite metal leads connected to the sample along the $x$ direction as shown in Fig. 1(a). To avoid redundant scattering from mismatched interface between the leads and central scattering region, the two leads are also modeled by clean Dirac semimetal thin films. In our numerical simulations, we set the chemical potentials of leads $\mu_{L, R}=100 \mathrm{meV}$ to guarantee a high density of states. We introduce the Anderson type disorders to the central scattering region through random on-site energy with a uniform distribution within
$[-U / 2, U / 2]$, with the disorder strength $U$. This setup allows us to calculate the longitudinal conductance $\sigma$ and the distribution of local current.

Fig. 2(b) and 2(e) show the conductance $\sigma$ of Dirac semimetal thin film with different thickness $\left(L_{z}=7 a_{z}\right.$ and $\left.5 a_{z}\right)$ versus disorder strength. The Fermi level $E_{f}=15 \mathrm{meV}$ is chosen inside the band gap caused by confinement effect. We found that the conductance keeps quantized without any fluctuations if the disorder strength $U$ is smaller than $U_{c}=1 \mathrm{eV}$ when the system is in the QSH regime $\left(L_{z}=7 a_{z}\right)$. The conductance plateau collapses when the disorder is strong enough. The quantized conductance plateau manifests the robustness of helical edge states to the weak disorder. Such observation agrees with the previous result in the literatures $\underline{49}-52$ While intriguing phenomena emerge in the normal state $\left(L_{z}=5 a_{z}\right)$. Initially, the conductance is zero since there is no electronic state inside the band gap for a normal insulator. With the increasing of the disorder strength, the conductance becomes finite and reaches to a quantized value $\left(\sigma=2 e^{2} / h\right)$, then the conductance maintains at this value for a certain range before eventually decreases. The absence of fluctuation in the quantized conductance plateau denotes that the quantized conductance is contributed by the helical edge states, indicating the topological nature of the TAI phenomena.

Fig. 2(c) and 2(f) show the phase diagram obtained by numerical simulations. Each point corresponds to a single realization of the disorder potential, which turns 
out to be sufficient for determining the region of TAI phase. We find that this quantized region appears not only in the band gap, but also occurs in a small region of the conduction bands $\left(L_{z}=7 a_{z}\right)$ and the valence bands $\left(L_{z}=5 a_{z}\right)$.

To substantiate the assertion that the quantized conductance plateau originates from the robust edge states, we study the nonequilibrium local current distribution between neighboring sites $\mathbf{i}$ and $\mathbf{j}$ from the formula 25

$$
J_{\mathbf{i} \rightarrow \mathbf{j}}=\frac{2 e^{2}}{h} \operatorname{Im}\left[\sum_{\alpha, \beta} H_{\mathbf{i} \alpha, \mathbf{j} \beta} G_{\mathbf{j} \beta, \mathbf{i} \alpha}^{n}\left(E_{f}\right)\right]\left(V_{L}-V_{R}\right),
$$

where $G^{n}\left(E_{f}\right)=G^{r} \Gamma_{L} G^{a}$ is electron correlation function. To calculate the local current distribution, a small external bias $V=V_{L}-V_{R}$ is applied longitudinally between the two terminals, where $V_{L}$ and $V_{R}$ describe the voltages of the left and right leads. We assume the electrostatic potential in the left lead to be $1 \mathrm{meV}$ and zero in the right lead. The electrostatic potential in the central part is $\phi\left(i_{x}\right)=\left(L_{x}-i_{x}+1\right) /\left(L_{x}+1\right) \mathrm{meV}$, where $i_{x}$ is the site index along the $x$-direction and $1 \leq i_{x} \leq L_{x}$. Then, the electric field is uniformly distributed in the central device region. As shown in Fig. 3, the red and blue arrows correspond to the strength of local currents of the upper and lower blocks, respectively. Apparently, the spin-up and spin-down local currents are localized at the two different side surfaces, respectively. The QSH insulator is characterized by the helical edge states shown in Fig. 3.

\section{BORN APPROXIMATION}

To corroborate the physical interpretation of numerical simulation, we analyze the present model within an effective medium theory based on the Born approximation in which high order scattering processes are neglected.26 In the self-consistent Born approximation, the self-energy $\Sigma$ for a finite disorder strength is given by the following integral equation

$$
\Sigma=\frac{U^{2}}{12}\left(\frac{a}{2 \pi}\right)^{2} \int_{1 B Z} d \mathbf{k}_{\|} \frac{1}{E_{f}-H\left(\mathbf{k}_{\|}\right)-\Sigma},
$$

where $H\left(\mathbf{k}_{\|}\right)$is the model Hamiltonian of Dirac semimetal with the confinement imposed along $z$ direction. The coeffcient $1 / 12$ originates from the variance $\left\langle U^{2}\right\rangle=U^{2} / 12$ of a random variable uniformly distributed in the range $[-U / 2, U / 2]$. This integration is over the first Brillouin zone (1BZ). We will use the lowestorder Born approximation, which means setting $\Sigma=0$ on the right hand side of Eq. (5).

When the confinement is imposed along $z$ direction with $L_{z}=5 a_{z}$, by calculating the bottom of the conduction band $\tilde{E}_{1}$ and the top of the valence band $\tilde{E}_{2}$ of the renormalized Hamiltonian $(\tilde{H}=H+\Sigma)$ as a function of $E_{f}$ and $U$, we obtain the red and green dashed

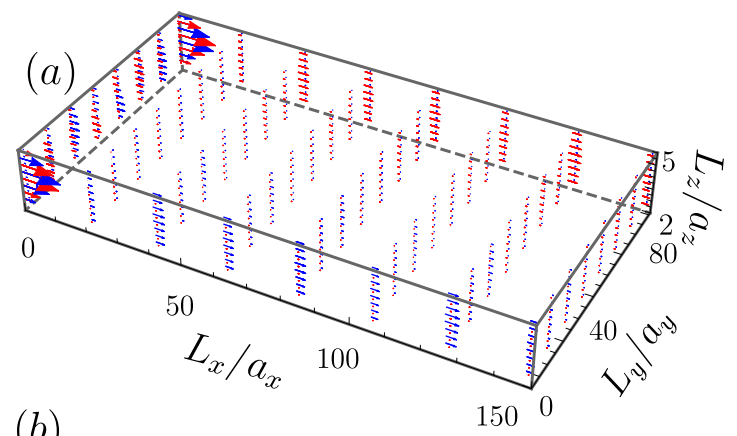

(b)

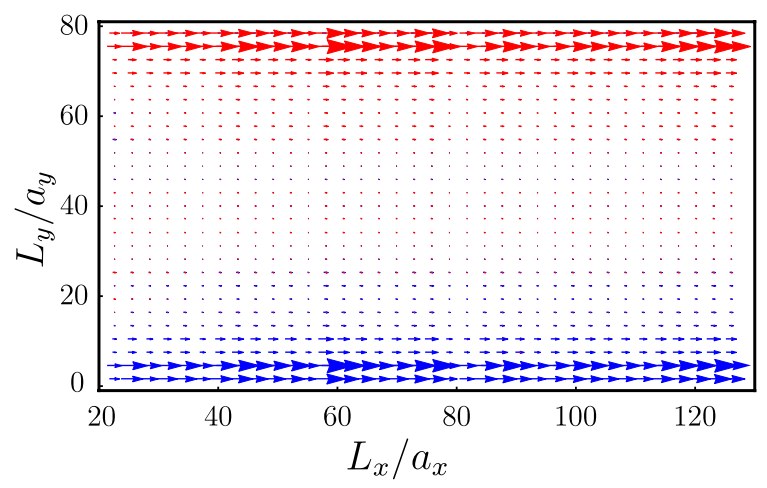

FIG. 3: (Color online) (a) The averaged nonequibrium local current distribution for the TAI phases of the Dirac semimetal thin film with thinkess scale $L_{z}=5 a_{z}$, Fermi level $E_{f}=$ $15 \mathrm{meV}$ and the disorder strength $U=1000 \mathrm{meV}$ in a two terminal setup. The red and blue arrows correspond to the current of the spin-up and spin-down component, respectively. The arrow size means the current strength. (b) The cross section of (a) with $L_{z}=3 a_{z}$.

curves in Fig. 2(f). The red curve denotes the phase boundary line $\tilde{E}_{1}-\tilde{E}_{2}=\Delta_{\tilde{E}}=0$, which means the gap closing. The region inside the green dashed lines is determined by $\tilde{E}_{2}<\tilde{E}_{f}<\tilde{E}_{1}$, which is another necessary condition for the TAI phenomena, i.e., the renormalized Fermi level must be inside the renormalized energy gaps. We find that the results based on Born approximation fit well with the previous numerical calculations, which means that disorder has a renormalized effect on bulk band gap $\Delta_{E}$ and the Fermi level $E_{f}$, leading to the TAI phenomena in Dirac semimetal thin films.

To demonstrate the disorder-induced topological phase transition, the spin Chern number $\tilde{C}_{s}$ of the renormalized Hamiltonian $\tilde{H}$ is also evaluated for various $U$ and $E_{f}$ by using the quantum well approximation. It is found that the topological mass $\mathcal{M}_{n}$ of each quantum well modes are renormalized by disorder. An approximate analytic solution of the effective mass $\tilde{\mathcal{M}}_{n}$ is given by ${ }^{26}$

$$
\tilde{\mathcal{M}}_{n}=\mathcal{M}_{n}-\frac{U^{2} a^{2}}{48 \pi} \frac{M_{2}}{M_{2}^{2}-C_{2}^{2}} \ln \left|\frac{M_{2}^{2}-C_{2}^{2}}{E_{f}^{2}-\mathcal{M}_{n}^{2}} \frac{\pi^{4}}{a^{4}}\right|,
$$

showing that the disorder correction to the topological mass $\mathcal{M}_{n}$ is negative, provided by $M_{2}>0$ and $\left|M_{2}\right|>\left|C_{2}\right|$. For a clean system with $L_{z}=5 a_{z}$, all 
of the subsystems are trivial, and this system is a normal insulator with $C_{s}=0$. For a certain disorder strength (about $750 \mathrm{meV}$ ), the first quantum well mode is inverted with a negative mass $\tilde{\mathcal{M}}_{1}<0$, and this system turns to QSH insulator with $\tilde{C}_{s}=1$.

\section{CONCLUSION}

In this paper, we investigate disorder-induced TAI in Dirac semimetal thin films. We observe a transition from a trivial insulating phase to a QSH state at a finite disorder strength. We present the phase diagram as a function of the disorder strength and the Fermi level, which is in accordance with the result obtained by the effective medium theory based on the Born approximation. We also plot the nonequilibrium local current distribution, which further confirms that the quantized conductance plateau in the TAI phase arises from the helical edge states induced by disorder.

\section{Acknowledgments}

This work was supported by the National Natural Science Foundation of China (Grant No. 11274102), the Program for New Century Excellent Talents in University of Ministry of Education of China (Grant No. NCET11-0960), and Specialized Research Fund for the Doctoral Program of Higher Education of China (Grant No. 20134208110001).
1 K. Novoselov, A. Geim, S. Morozov, D. Jiang, Y. Zhang, S. Dubonos, I. Grigorieva, and A. Firsov, Science 306, 666 (2004).

2 A. H. Castro Neto, F. Guinea, N. M. R. Peres, K. S. Novoselov, and A. K. Geim, Rev. Mod. Phys. 81, 109 (2009).

3 V. N. Kotov, B. Uchoa, V. M. Pereira, F. Guinea, and A. H. Castro Neto, Rev. Mod. Phys. 84, 1067 (2012).

4 J. E. Moore, Nature (London) 464, 194 (2010).

${ }^{5}$ M. Z. Hasan and C. L. Kane, Rev. Mod. Phys. 82, 3045 (2010).

6 X.-L. Qi and S.-C. Zhang, Rev. Mod. Phys. 83, 1057 (2011).

7 Z. Wang, Y. Sun, X. Q. Chen, C. Franchini, G. Xu, H. Weng, X. Dai, and Z. Fang, Phys. Rev. B 85, 195320 (2012).

8 Z. Wang, H. Weng, Q. Wu, X. Dai, and Z. Fang, Phys. Rev. B 88, 125427 (2013).

9 Z. K. Liu, B. Zhou, Y. Zhang, Z. J. Wang, H. Weng, D. Prabhakaran, S.-K. Mo, Z. X. Shen, Z. Fang, X. Dai, Z. Hussain, and Y. L. Chen, Science 343, 864 (2014).

10 Z. K. Liu, J. Jiang, B. Zhou, Z. J. Wang, Y. Zhang, H. M. Weng, D. Prabhakaran, S.-K. Mo, H. Peng, P. Dudin, T. Kim, M. Hoesch, Z. Fang, X. Dai, Z. X. Shen, D. L. Feng, Z. Hussain, and Y. L. Chen, Nat. Mater. 13, 677 (2014).

11 M. Neupane, S.-Y. Xu, R. Sankar, N. Alidoust, G. Bian, C. Liu, I. Belopolski, T.-R. Chang, H.-T. Jeng, H. Lin, et al., Nat. Commun. 5, 3786 (2014).

12 B. J. Yang and N. Nagaosa, Nat. Commun. 5, 4898 (2014).

13 S. Y. Xu, C. Liu, S. K. Kushwaha, R. Sankar, J. W. Krizan, I. Belopolski, M. Neupane, G. Bian, N. Alidoust, T.-R. Chang, H.-T. Jeng, C.-Y. Huang, W.-F. Tsai, H. Lin, P. P. Shibayev, F.-C. Chou, R. J. Cava, and M. Z. Hasan, Science 347, 294 (2015).

14 J. Xiong, S.K. Kushwaha, T. Liang, J. W. Krizan, M. Hirschberger, W. Wang, R. J. Cava, and N. P. Ong, Science 350, 413 (2015).

15 B. Zhou, H. Z. Lu, R. L. Chu, S. Q. Shen, and Q. Niu, Phys. Rev. Lett. 101, 246807 (2008).

16 J. Linder, T. Yokoyama, and A. Sudbo, Phys. Rev. B 80, 205401 (2009).

17 Y. Zhang, K. He, C. Z. Chang, C. L. Song, L. L. Wang, X.
Chen, J. F. Jia, Z. Fang, X. Dai, W. Y. Shan, S. Q. Shen, Q. Niu, X. L. Qi, S. C. Zhang, X. C. Ma, and Q. K. Xue, Nat. Phys. 6, 584 (2010).

18 H. Z. Lu, W. Y. Shan, Y. Wang, Q. Niu, and S. Q. Shen, Phys. Rev. B 81, 115407 (2010).

19 C. X. Liu, H. J. Zhang, B. Yan, X. L. Qi, T. Frauenheim, X. Dai, Z. Fang, and S. C. Zhang, Phys. Rev. B 81, 041307 (2010).

20 K. I. Imura, M. Okamoto, Y. Yoshimura, Y. Takane, and T. Ohtsuki, Phys. Rev. B 86, 245436 (2012).

21 X. Xiao, S. A. Yang, Z. Liu, H. Li, and G. Zhou, Sci. Rep. 5, 7898 (2015).

${ }^{22}$ H. Pan, M. Wu, Y. Liu, and S. A. Yang, Sci. Rep. 5, 14639 (2015).

23 P. A. Lee and T. V. Ramakrishnan, Rev. Mod. Phys. 57, 287 (1958).

24 J. Li, R. L. Chu, J. K. Jain, and S. Q. Shen, Phys. Rev. Lett. 102, 136806 (2009).

25 H. Jiang, L. Wang, Q.-F. Sun, and X. C. Xie, Phys. Rev. B 80, 165316 (2009).

26 C. W. Groth, M. Wimmer, A. R. Akhmerov, J. Tworzydło, and C. W. J. Beenakker, Phys. Rev. Lett. 103, 196805 (2009).

27 Y. X. Xing, L. Zhang, and J. Wang, Phys. Rev. B 84, 035110 (2011).

28 C. P. Orth, T. Sekera, C. Bruder, and T. L. Schmidt, Sci. Rep. 6, 24007 (2016).

29 H. M. Guo, G. Rosenberg, G. Refael, and M. Franz, Phys. Rev. Lett. 105, 216601 (2010).

30 H. Guo, S. Feng, and S. Q. Shen, Phys. Rev. B 83, 045114 (2011).

31 H. M. Guo, Phys. Rev. B 82, 115122 (2010).

32 B. Wu, J. Song, J. Zhou, and H. Jiang, Chin. Phys. B 11, 117311 (2016).

33 J. Song, H. Liu, H. Jiang, Q. F. Sun, and X. C. Xie, Phys. Rev. B 85, 195125 (2012).

34 A. Girschik, F. Libisch, and S. Rotter, Phys. Rev. B 88, 014201 (2013).

35 A. A. Burkov and L. Balents, Phys. Rev. Lett. 107, 127205 (2011).

36 K. Kobayashi, Y. Yoshimura, K.-I. Imura, and T. Ohtsuki, Phys. Rev. B 92, 235407 (2015). 
37 Y. Yoshimura, W. Onishi, K. Kobayashi, T. Ohtsuki, and K.-I. Imura, Phys. Rev. B 94, 235414 (2016).

38 B. A. Bernevig, T. L. Hughes, and S. C. Zhang, Science 314, 1757 (2006).

39 D. N. Sheng, Z. Y. Weng, L. Sheng, and F. D. M. Haldane, Phys. Rev. Lett. 97, 036808 (2006).

40 H. Li, L. Sheng, D. N. Sheng, and D. Y. Xing, Phys. Rev. B 82, 165104 (2010).

41 E. Prodan, Phys. Rev. B 80, 125327 (2009).

42 T. Fukui and Y. Hatsugai, Phys. Rev. B 75, 121403(R) (2007).

${ }^{43}$ Y. Yang, Y. F. Zhang, L. Sheng, and D. Y. Xing, Europhys. Lett. 105, 27005 (2014).

44 R. Landauer, Philos. Mag. 21, 863 (1970).

45 M. Büttiker, Phys. Rev. B 38, 9375 (1988).
46 D. S. Fisher and P. A. Lee, Phys. Rev. B 23, 6851 (1981).

47 A. MacKinnon, Z. Phys. B-Condensed matter 59, 385 (1985).

48 G. Metalidis, and P. Bruno, Phys. Rev. B 72, 235304 (2005).

49 C. L. Kane and E. J. Mele, Phys. Rev. Lett. 95, 226801 (2005).

50 C. L. Kane and E. J. Mele, Phys. Rev. Lett. 95, 146802 (2005).

51 L. Sheng, D. N. Sheng, C. S. Ting, and F. D. M. Haldane, Phys. Rev. Lett. 95, 136602 (2005).

52 A. A. Taskin, S. Sasaki, K. Segawa, and Y. Ando, Phys. Rev. Lett. 109, 066803 (2012). 